\title{
Amplitude suppression and chaos control in epileptic EEG signals
}

\author{
KAUSHIK MAJUMDAR* and MARK H. MYERS \\ Department of Computer Science, University of Memphis, Memphis, TN 38152, USA
}

(Revised 26 May 2006; accepted 5 June 2006)

\begin{abstract}
In this paper we have proposed a novel amplitude suppression algorithm for EEG signals collected during epileptic seizure. Then we have proposed a measure of chaoticity for a chaotic signal, which is somewhat similar to measuring sensitive dependence on initial conditions by measuring Lyapunov exponent in a chaotic dynamical system. We have shown that with respect to this measure the amplitude suppression algorithm reduces chaoticity in a chaotic signal (EEG signal is chaotic). We have compared our measure with the estimated largest Lyapunov exponent measure by the largelyap function, which is similar to Wolf's algorithm. They fit closely for all but one of the cases. How the algorithm can help to improve patient specific dosage titration during vagus nerve stimulation therapy has been outlined.
\end{abstract}

Keywords: Epileptic EEG signal; Chaotic signal; Amplitude suppression; Chaos control; Average signal Lyapunov exponent (ASLE); Vagus nerve stimulation (VNS)

\section{Introduction}

Amplitude suppression in a signal may have important applications, for example, it has been shown that off line spike suppression in a sampled EEG signal can help to determine dosage titration in electrical stimulation therapy to control epileptic seizures [1]. By spike we mean very high amplitude phases of an EEG signal. During epileptic seizure neurons fire in unusual synchrony and this gives rise to heightened action potentials, which is manifested in the form of spikes in the EEG signal. In this paper we shall describe a novel algorithm, which is able to suppress amplitude in an epileptic EEG signal. The beauty of the algorithm is that it only suppresses the amplitude at each and every point by the desired (predetermined) amount and does nothing else. All other characteristics of the signal remain exactly the same. In other words unlike filtering we do not lose any information.

Chaos control has been a hot topic of research for the last fifteen years, since the seminal paper of Ott, Grebogy and Yorke appeared [2]. Numerous applications of chaos control have been found both in science and engineering [3]. Many systems of interest in science and engineering are dynamical systems, that is, systems, which change with respect to time. Roughly speaking, a chaotic dynamical system is one, which cannot be predicted with good accuracy. A rigorous mathematical definition of chaos appears in reference [4]. A subsequent modification of the definition appeared in [5]. A time series output of a dynamical system

*Corresponding author. Email: mkkaushik@hotmail.com

Computational and Mathematical Methods in Medicine

ISSN 1748-670X print/ISSN 1748-6718 online (c) 2006 Taylor \& Francis

http://www.tandf.co.uk/journals

DOI: $10.1080 / 10273660600890032$ 
is called a signal. When the dynamical system is chaotic we call the output signal chaotic. A brain is considered a chaotic dynamical system $[6,7]$ and therefore the EEG signals, produced by it, are chaotic in general. A signal can be chaotic in another sense-when the amplitude is a random variable with respect to time. EEG signals are chaotic in that sense too [8]. In this paper we have proposed a measure of chaoticity for a chaotic signal. With respect to this measure our amplitude suppression algorithm reduces chaoticity in a chaotic signal.

In section 2 we describe the amplitude suppression algorithm for epileptic EEG signals. In section 3 we describe the new measure of chaoticity in a chaotic signal and show how the amplitude suppression algorithm is reducing chaoticity in a chaotic signal. We call this new measure average signal Lyapunov exponent (ASLE). In section 4 we have compared ASLEs of ten different EEG signals collected during epileptic seizure with estimated largest Lyapunov exponents as calculated by the largelayap function [9]. They fit closely with each other in all but one channel. This suggests that ASLE is a good measure of chaoticity. In section 5 we have outlined a new stimulation protocol for the dosage titration in vagus nerve stimulation (VNS) based on the spike suppression algorithm.

\section{Amplitude suppression in epileptic EEG signals}

In this section we shall describe a novel algorithm to suppress the amplitude of an EEG signal, collected from an epileptic patient during seizure, keeping all other characteristics of the signal intact. We shall first plot the signal in the Euclidean plane in order to treat it like a trajectory of a dynamical system with a two-dimensional (2D) phase space. Then we introduce new parameters into the system, in order to use them as control parameters. Then we plot the trajectory with suitable parameter values and transform back that trajectory into a "signal", which is a mirror image of the original signal except for reduced amplitude at each time instant. Throughout this paper unless otherwise mentioned, $r=r(t)$ will denote amplitude of a signal at time $t$. The signal is actually sampled and hence digital. $t$ takes integer values only.

1. Proc (Amplitude_suppression)

2. Input = An epileptic EEG signal in time domain;

3. Output $=$ Exactly the same signal except for reduced amplitude at each time instant;

4. Choose $(a, b)>(0,0)$;

5. For $t=$ first sample point to the last sample point

6. $\quad\{\mathbf{P l o t}(\operatorname{ar}(t) \cos (t), b r(t) \sin (t))$;

7. $\quad$ Add two subsequent points by a line; $\}$

8. End

9. For $(x, y)=$ first point to last point

10. $\quad\left\{\boldsymbol{P l o t}\left(t=\arctan (a y / b x), r=\left(x^{2}+y^{2}\right)^{1 / 2}\right)\right.$;

11. Add two subsequent points by a line; $\}$

12. End

13. Proc (Choose $(a, b))$;

14. $a>0$;

15. $b>0$;

16. $a \neq b$;

17. $a+b=1$; 
Clearly, if we take $a+b=1$ then $0<a<1$ and $0<b<1$. Both $x$ and $y$ will be smaller after dampening (suppression). So amplitude will be smaller. Since $a+b=1$, taking a smaller value for $a$ means taking a larger value for $b$ and vice versa. This keeps the whole dampening in nice balance. For a near uniform reduction in amplitudes over all the time instants it is recommended that $a$ should be taken slightly $<0.5$ and $b$ should be taken slightly $>0.5$.

Notice that the total amount of reduction in amplitude of the EEG signal is

$$
\Delta r=r(t) \sqrt{(1-a)^{2} \cos ^{2} t+(1-b)^{2} \sin ^{2} t} .
$$

If $\Delta r / r$ (when $r \neq 0$ ) is to be made constant, i.e. the change in signal is uniformly $(\Delta r / r) \times 100 \%$ across all $t$ then $(\mathrm{d} f(t) / \mathrm{d} t)=0$, where $f(t)=(1-a)^{2} \cos ^{2} t+(1-b)^{2} \sin ^{2}(t)$. This implies

$$
(a-b)(a+b-2) \sin 2 t=0
$$

for all $t$. That is, either

$$
a=b,
$$

or,

$$
a+b=2 .
$$

Equation (2.4) will actually enhance the amplitude of the signal unless $a=b=1$, in which case the original signal will remain unchanged. From equation (2.1) it is clear that when equation $(2.3)$ holds the signal after dampening is changed by $(1-a) \times 100 \%$. To avoid too much dampening to the signal, when that is required, it is recommended that $a$ be chosen $>0.5$. In light of this we can rewrite the subroutine Choose in the following manner.

\section{Proc (Choose $(a, b))$}

19. $a>0$

20. $b>0$

21. $a=b>0.5$;

Note that when $a=b=0.6$ the spike suppression is by $40 \%$, when $a=b=0.7$ the spike suppression is by $30 \%$, etc. This may potentially be used to regulate the amplitude suppression uniformly by a fixed percentage. So we have come up with two spike suppression algorithms for a signal, one in variable mode and the other in fixed mode.

Now we shall present a human epileptic EEG data based example to demonstrate the method described so far. In figure 1 raw EEG signal collected through a single on the scalp electrode (out of seventeen such electrodes placed all over the scalp of an epileptic patient) during the onset of a seizure, has been shown. Amplitude of the signal at certain instant becomes much higher than usual, which is called spike. The same EEG signal has been plotted by the absolute value of the amplitude in figure 2. From figure 2 it appears that if we can reduce the spike amplitude by $(1-(6 / 8)) \times 100=25 \%$ (say, by applying electrical stimulation to the brain [10]) then that will be able to control the seizure [11]. So according to the fixed mode spike suppression (i.e. $a=b=(6 / 8)$ ) the brain will have to be stimulated in a way to produce $25 \%$ across the board amplitude 
reduction. In the next section we shall calculate the amount of stimulation in such a situation.

Figure 3 through 9 describe the algorithm and its result on a particular EEG signal. The signal of figure 1 has been polar-plotted in figure 3. Parameters $a$ and $b$ have been introduced into the polar-plot and this has been shown in figure 4. Figure 4 shows the amplitude suppression by introducing new parameters and carefully choosing their values (between 0 and 1). Figure 4 describes lines 5 through 8 in the algorithm. In all the figures suppressed signal has been shown in blue and non-suppressed one in red. Figure 5 gives the signal in absolute value of the amplitude as got back by inverse transformation from figure 4. Figure 5 shows lines 9 through 12 of the algorithm. Figure 6 shows the comparison result. It shows the original signal (in red as shown in figure 1) superimposed with the suppressed signal (in blue as shown in figure 5). The superimposition is on every time instant and both the signals are plotted in absolute value of amplitude. Figure 7 depicts in blue the difference in amplitude at each time instant between the original and the suppressed signal. It is the plot of $\Delta r(t)$ as given by equation (2.1). Figures 8 and 9 give the superimposition of $\Delta r(t)$ (in blue) on the original signal (in red) for two different set of parameter values. These two figures comparatively show why the values of $a$ and $b$ should be taken close to 0.5 in order to keep the amount of suppression moderate.

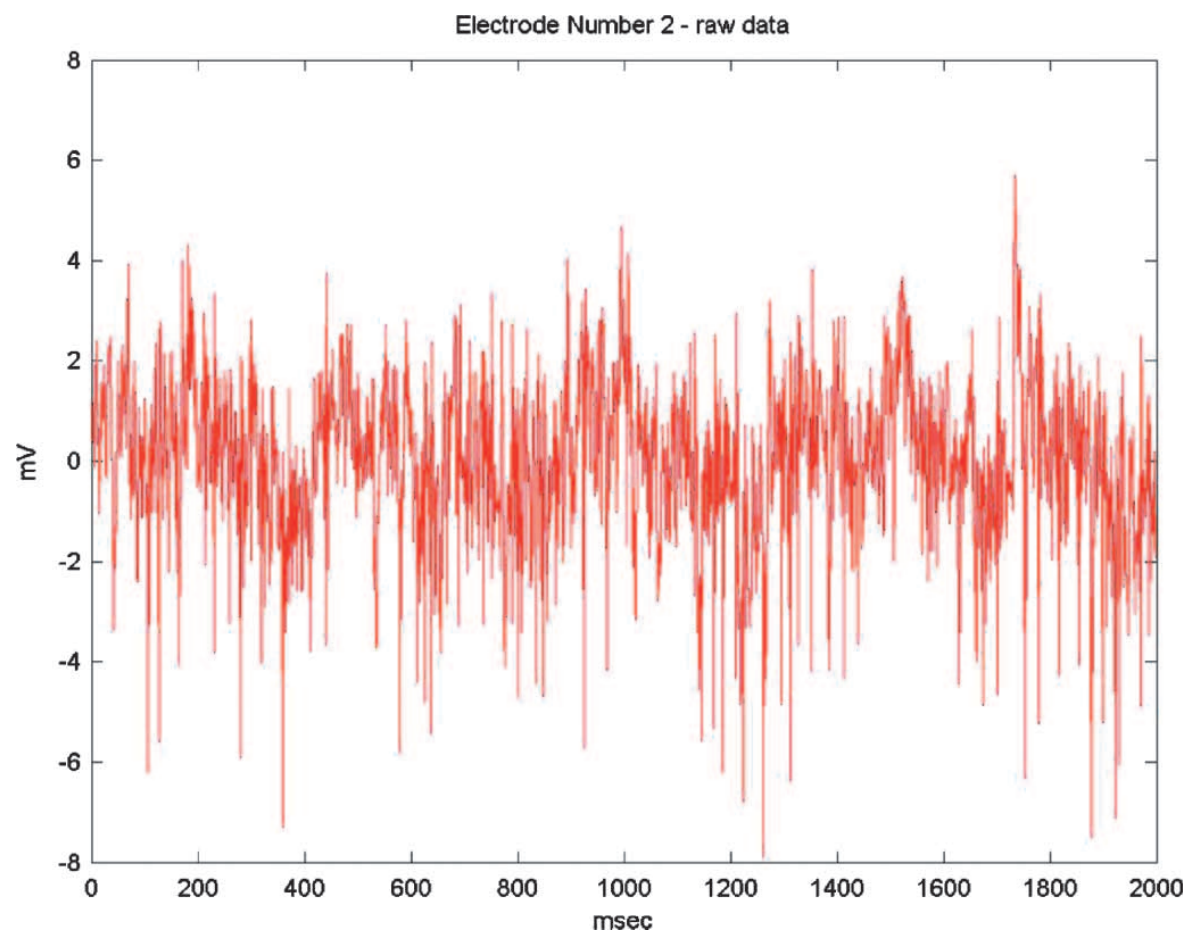

Figure 1. Millivolt (mV) vs. millisecond (msec) plot of the raw EEG signal collected through electrode number 2 (channel 2) out of a total 17 electrodes placed over the scalp of an epileptic patient during the occurrence of a seizure. 


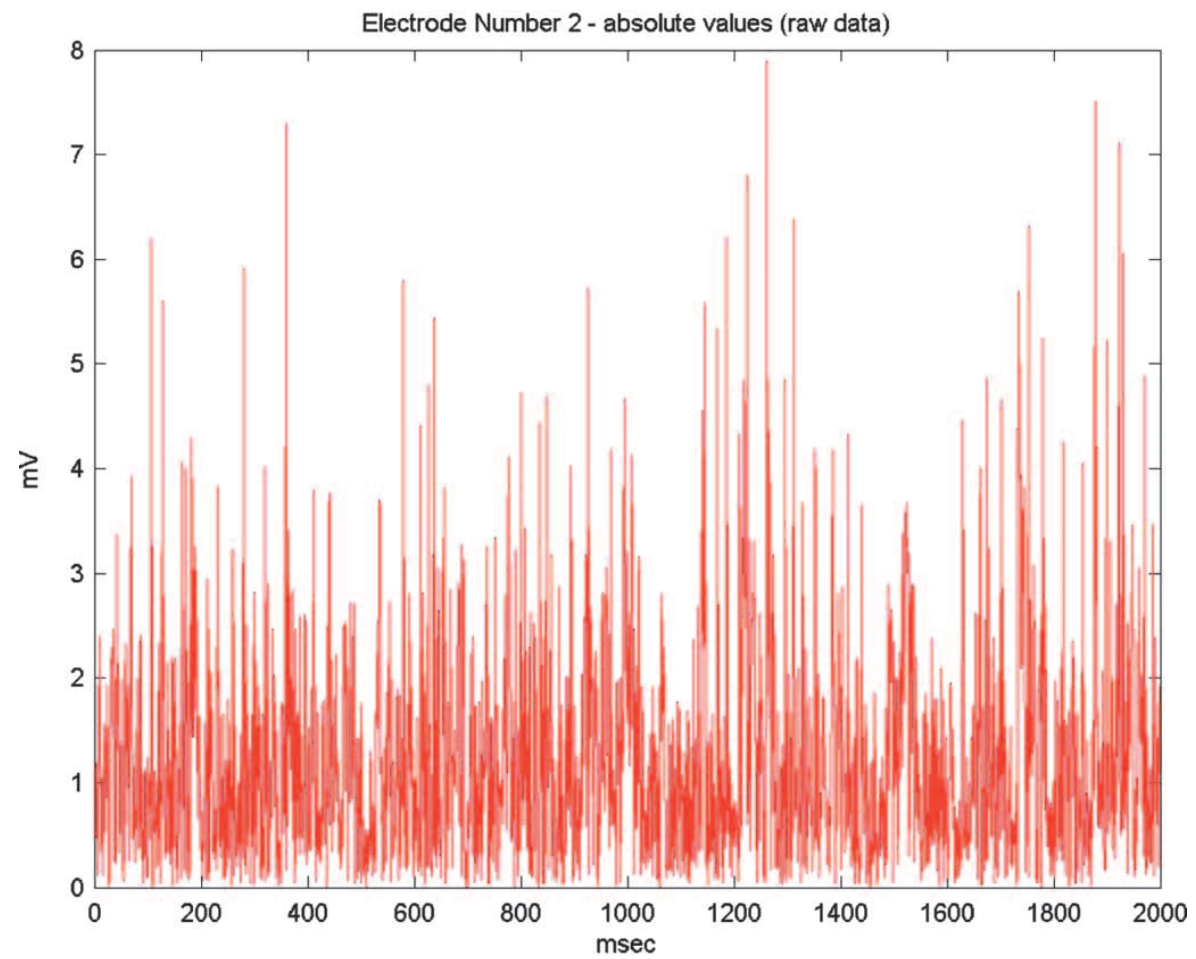

Figure 2. Plot of the absolute value of the amplitudes of the EEG signal shown in figure 1.

\section{Chaos control in EEG signals}

Despite widespread popularity of chaos control techniques and the abundance of highly random, erratic signals like EEG, not much attention has so far been paid to reduce the randomness in a signal by a chaos control methodology. Chaos has a precise mathematical definition [4], which is completely deterministic. According to this definition a system must satisfy three conditions (not independent [5]) to be chaotic. One of them is the sensitive dependence on initial conditions. This is the criterion almost universally adopted as the notion for chaoticity, i.e. any system, which shows sensitive dependence on initial conditions is recognized as chaotic. This condition is typically determined by measuring Lyapunov exponents [3]. For a multidimensional system there will be more than one Lyapunov exponents (one for each dimension). In a system with sensitive dependence on initial conditions, or chaotic system, at least one of them will have to be positive. So we can say that the largest Lyapunov exponent in a chaotic system is positive. In fact the value of the largest Lyapunov exponent can be taken as a measure of chaoticity of the system. The higher the value the more chaotic the system is.

To measure the sensitive dependence on initial conditions by the largest Lyapunov exponent the underlying dynamical system must have more than one trajectories, for the sensitive dependence on initial conditions is a measure of how two trajectories are (exponentially) moving apart from each other over time. But the polar plot of an EEG signal has only one trajectory. Here the chaoticity is indicated by how arbitrarily the trajectory is 


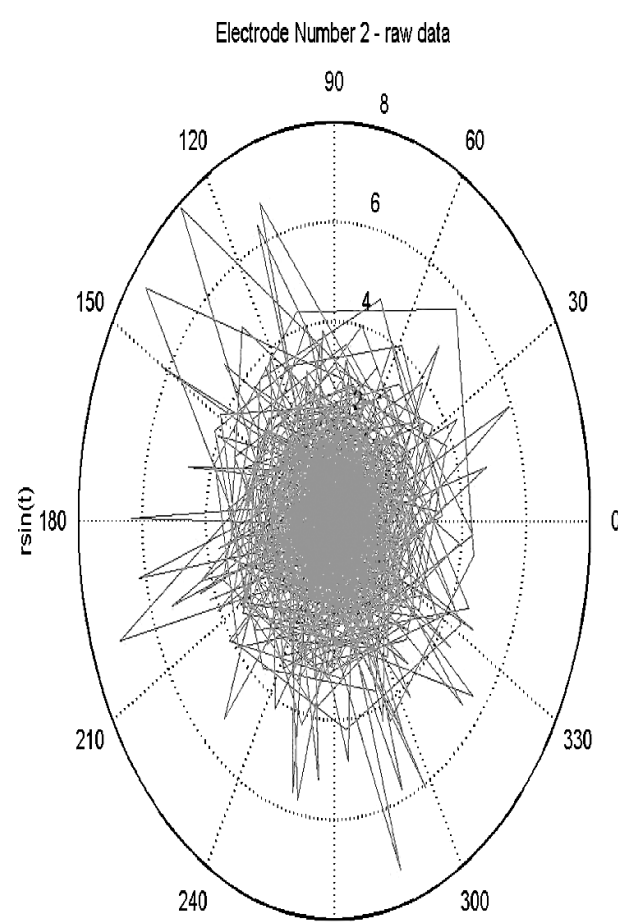

270

$\operatorname{rcos}(t)$

Figure 3. Polar plot of the original signal, where $r \cos (t)$ and $r \sin (t)$ are the projections along $X$ and $Y$ axes, respectively.

stretching or compressing during evolution over time (figures 3 and 4). Since the trajectory is 2D the stretching and compressing are taking place along $X$ and $Y$ axes. To measure the average stretching and compressing along each direction let us introduce a Lyapunov exponent like measure, which we may call average signal Lyapunov exponent (ASLE), which we define as follows, where $k$ is the number of data points.

$$
\begin{aligned}
& \lambda_{x}=\frac{1}{k} \sum_{i=1}^{k} \log \frac{\left|r\left(t_{i}+\Delta t_{i}\right) \cos \left(t_{i}+\Delta t_{i}\right)-r\left(t_{i}\right) \cos \left(t_{i}\right)\right|}{\Delta t_{i}} \\
& \lambda_{y}=\frac{1}{k} \sum_{i=1}^{k} \log \frac{\left|r\left(t_{i}+\Delta t_{i}\right) \sin \left(t_{i}+\Delta t_{i}\right)-r\left(t_{i}\right) \sin \left(t_{i}\right)\right|}{\Delta t_{i}} .
\end{aligned}
$$

The entire polar plot of a signal (figure 3 ) is like a phase space of a 2D dynamical system. It will have two ASLE $\lambda_{x}$ and $\lambda_{y}$, respectively along $X$ and $Y$ directions. In equations (3.1) and (3.2) we have defined the ASLE of the system, which is somewhat similar to calculating the conventional Lyapunov exponents [3]. Here we have used $\lambda_{x}$ and $\lambda_{y}$ to indicate how the amplitude suppression algorithm reduces their value and thereby controls chaos in a signal. 


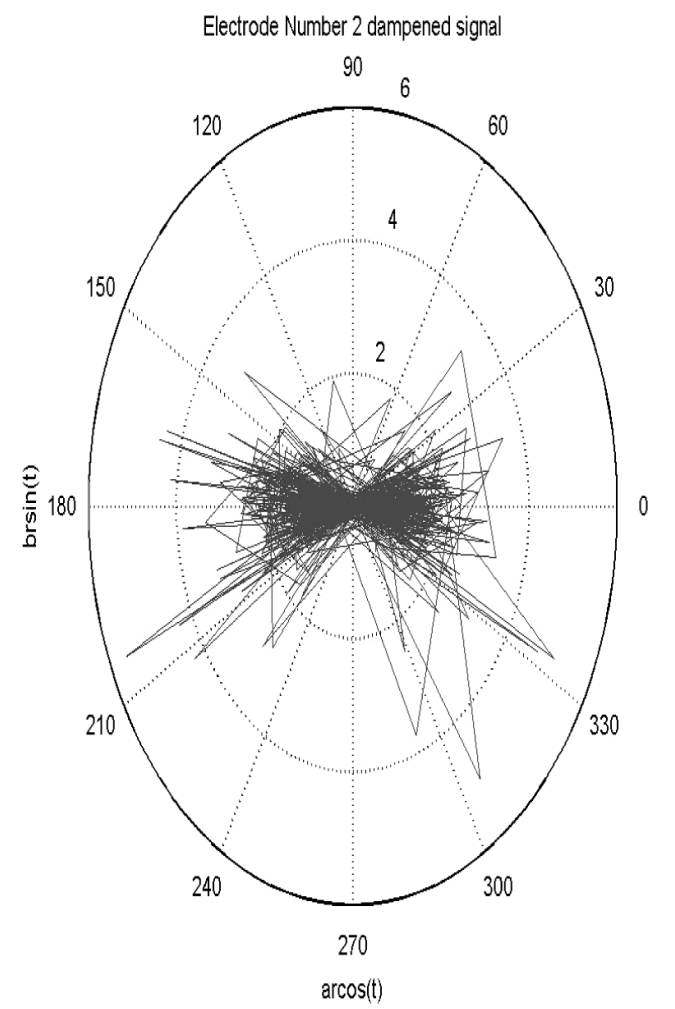

Figure 4. The polar plot with suppression by $a$ and $b$, where $a=0.8$ and $b=0.2$.

When amplitudes have been suppressed with $a$ and $b$ then equations (3.1) and (3.2) become

$$
\begin{aligned}
\lambda_{x} & =\frac{1}{k} \sum_{i=1}^{k} \log \frac{a\left|r\left(t_{i}+\Delta t_{i}\right) \cos \left(t_{i}+\Delta t_{i}\right)-r\left(t_{i}\right) \cos \left(t_{i}\right)\right|}{\Delta t_{i}} \\
& =\frac{1}{k} \sum_{i=1}^{k} \log \frac{\left|r\left(t_{i}+\Delta t_{i}\right) \cos \left(t_{i}+\Delta t_{i}\right)-r\left(t_{i}\right) \cos \left(t_{i}\right)\right|}{\Delta t_{i}}+\log \left(\frac{a}{\Delta t_{i}}\right) . \\
\lambda_{y} & =\frac{1}{k} \sum_{i=1}^{k} \log \frac{b\left|r\left(t_{i}+\Delta t_{i}\right) \sin \left(t_{i}+\Delta t_{i}\right)-r\left(t_{i}\right) \sin \left(t_{i}\right)\right|}{\Delta t_{i}} \\
& =\frac{1}{k} \sum_{i=1}^{k} \log \frac{\left|r\left(t_{i}+\Delta t_{i}\right) \sin \left(t_{i}+\Delta t_{i}\right)-r\left(t_{i}\right) \sin \left(t_{i}\right)\right|}{\Delta t_{i}}+\log \left(\frac{b}{\Delta t_{i}}\right) .
\end{aligned}
$$

$\Delta t_{i}=\Delta t_{j} \geq 1 \forall i, j$ and $0<a<1,0<b<1$, which imply that $\log \left(a / \Delta t_{i}\right)<0$ and $\log \left(b / \Delta t_{i}\right)<0$. That is, both the ASLE along $X$ and $Y$ axes have been reduced. $\lambda_{x}$ has been reduced by $\log \left(a / \Delta t_{i}\right)$ and $\lambda_{y}$ has been reduced by $\log \left(b / \Delta t_{i}\right)$. Particularly the largest ASLE, if it is positive, its absolute value is reduced. This means that the system has 


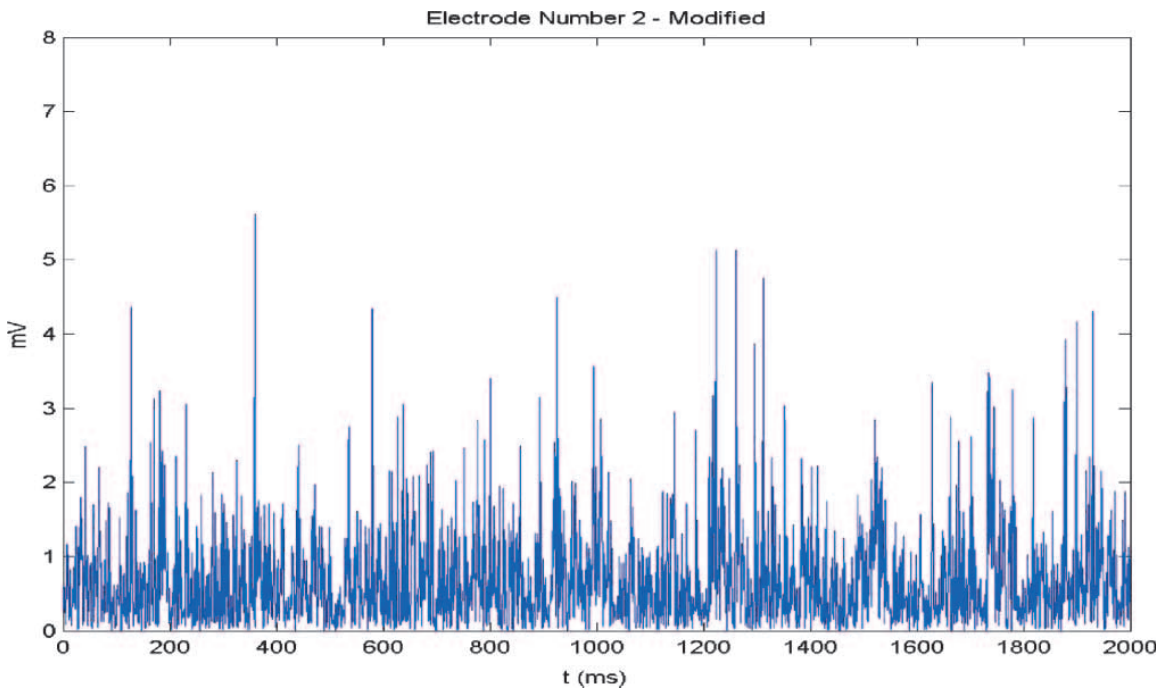

Figure 5. The damped signal. Only the absolute values of the amplitudes have been taken.

become less dependent on the initial conditions in the sense that after amplitude suppression if initially the stretching is small after some time the stretching may still be quite large, but not as large as in case of the original signal. Or, in other words it has become less chaotic. So our amplitude suppression algorithm also controls chaoticity in a signal. It is obvious from equations (3.3) and (3.4) that the arguments of the logarithmic functions will be fractions in case the signal is regular and this will produce negative

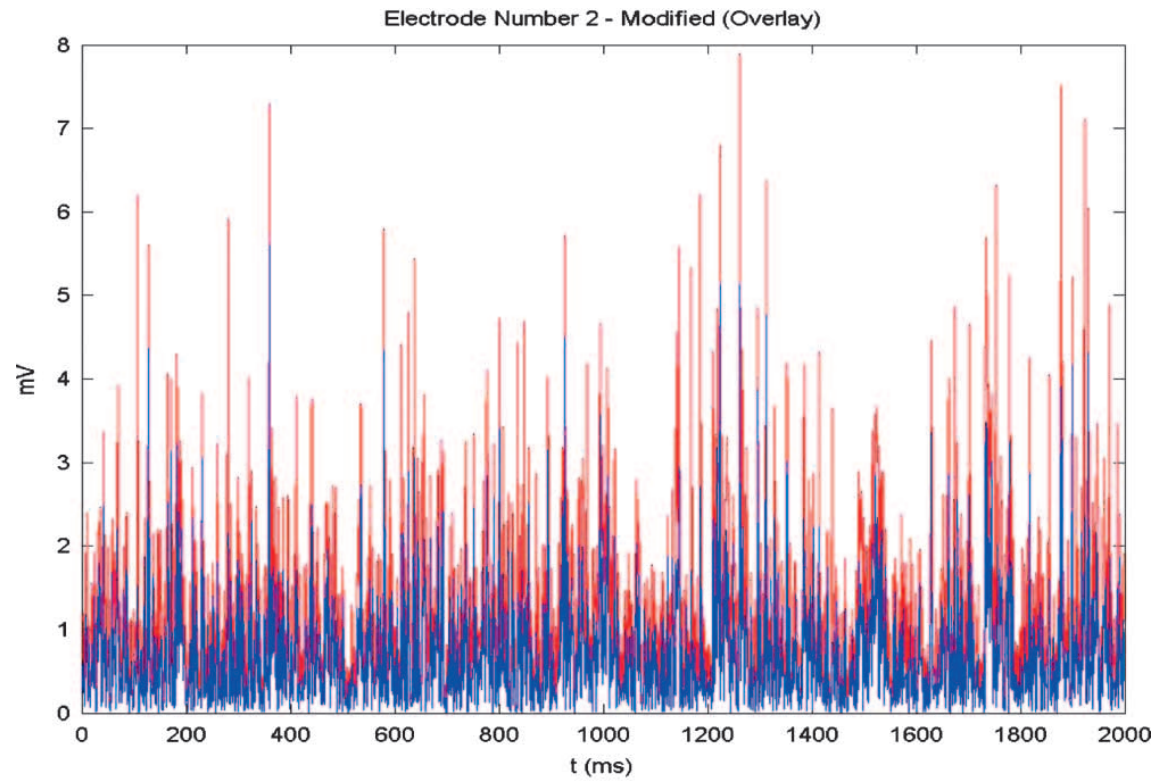

Figure 6. The superposition of the damped signal (blue) over the original one (red). Only the absolute values of the amplitudes have been taken. 


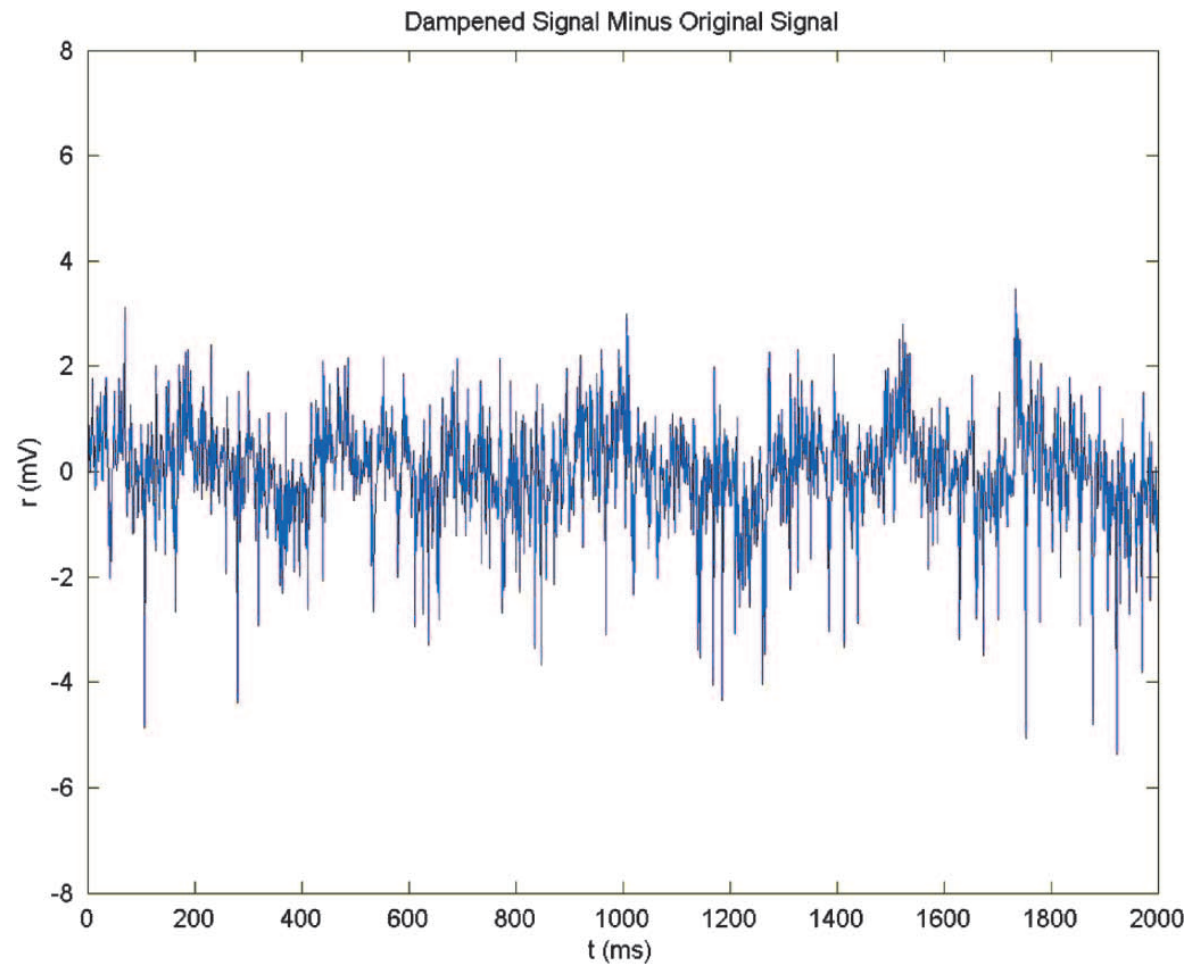

Figure 7. The difference of amplitudes between original signal and the damped one.

values for both $\lambda_{x}$ and $\lambda_{y}$. Notice that the signal is chaotic, but reduction of chaoticity depends on the amount of suppression induced and not on the signal. This is the beauty of the algorithm.

\section{ASLE vs. largest Lyapunov exponent}

Sensitive dependence on initial conditions has remained the most widely used criterion to determine if a dynamical system is chaotic. An $n$-dimensional dynamical system has $n$ different Lyapunov exponents, one for each dimension. A dynamical system to have sensitive dependence on initial conditions, and therefore to be chaotic, must have at least one positive Lyapunov exponent. The largest Lyapunov exponent of a dynamical system is a very reliable measure of chaoticity of the system. The largest Lyapunov exponent of a system can be calculated in several ways. Here we have followed the largelyap algorithm as described in [9], which is very similar to Wolf's algorithm [12]. We have run both ASLE and largelyap on ten different human epileptic EEG signals (EEG signals are considered chaotic [8]) collected through ten on the scalp electrodes from a patient during the onset of seizures. The results have been presented in table 1 .

Since ASLE treats a signal as a trajectory of a 2D dynamical system it gives two measures of stretching and/or compressing, one each in the direction of a coordinate axis. In table 1 we have printed both the values of ASLE. Only the larger one is to be taken as a measure of chaoticity and is to be compared with the largest Lyapunov exponent. Notice that for all 


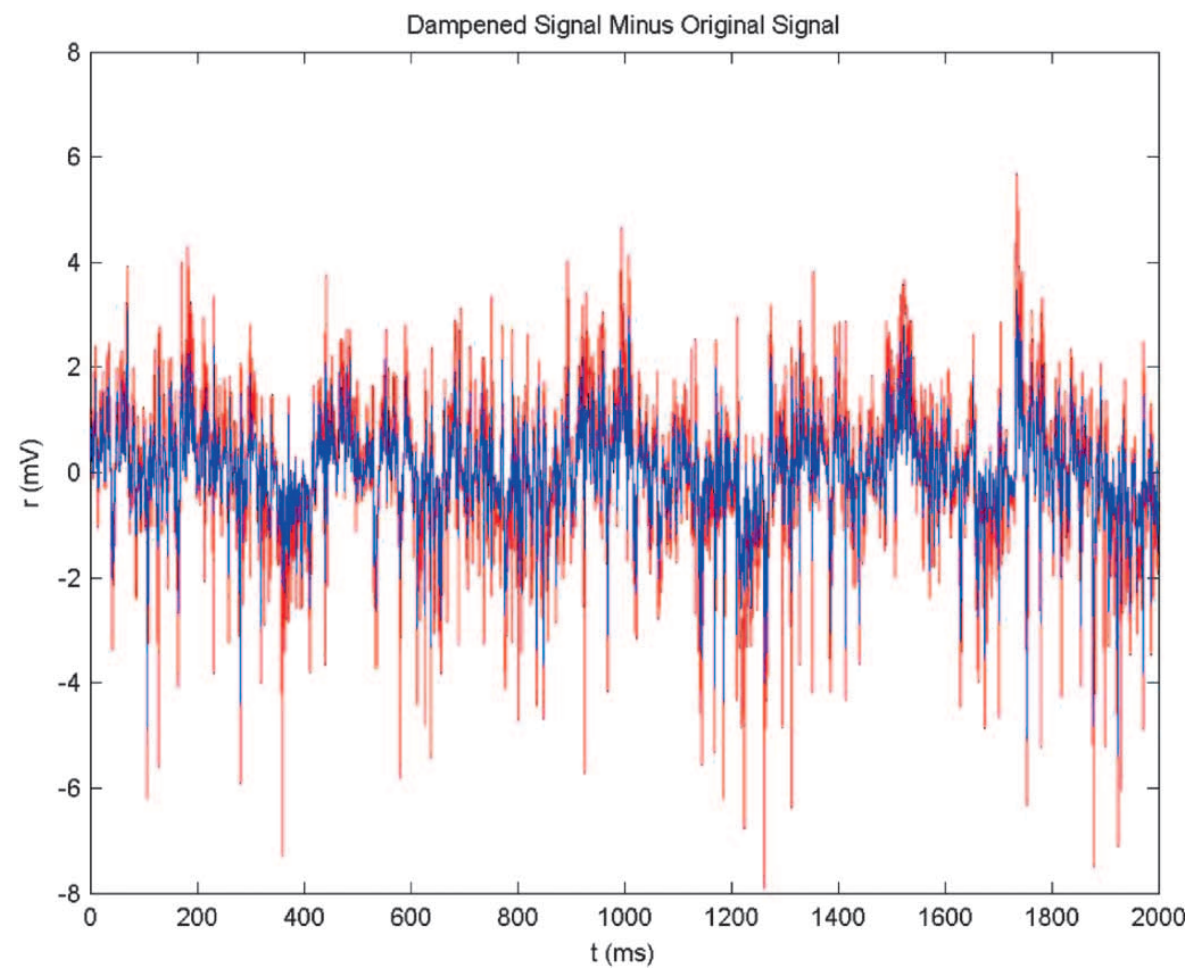

Figure 8. Superimposition of the difference between the original and the damped signals (blue) over the original one (red) for $a=0.8$ and $b=0.2$.

channels except one (channel 10) the largest ASLE value is quite close to the value determined by largelyap. In channel 10 the largelyap value is significantly less than that of the largest ASLE value of the EEG signal. However the prediction errors [9] of channel 10 remain quite high (e.g. higher than those of channel 9) and therefore the channel $10 \mathrm{EEG}$ signal is more chaotic (than, for example, channel 9), yet its largelyap value is low (e.g. lower than the EEG signal of channel 9). On the other hand the largest ASLE value gives a more consistent estimate of chaoticity (channel 9 value is less than channel 10). From this we can guess that the largest ASLE value is a relatively better measure of chaoticity in a chaotic signal than the largest Lyapunov exponent as estimated by largelyap (for the details of largelyap calculation and prediction error please see [9]).

\section{Possible effect on stimulation therapy}

In this section we will only briefly outline an approach to improve electrical stimulation therapy for the epileptic patients. However the approach is completely theoretical and no clinical trial is possible at this stage.

Electrical stimulation therapy has proved to be effective for a certain percentage of epileptic patients who do not respond to anticonvulsant drugs (for a review of various stimulation therapies for the epileptic patients see [13]). It is often tried as a better alternative before surgical resection, whose side effects may be significant. How various stimulations, 


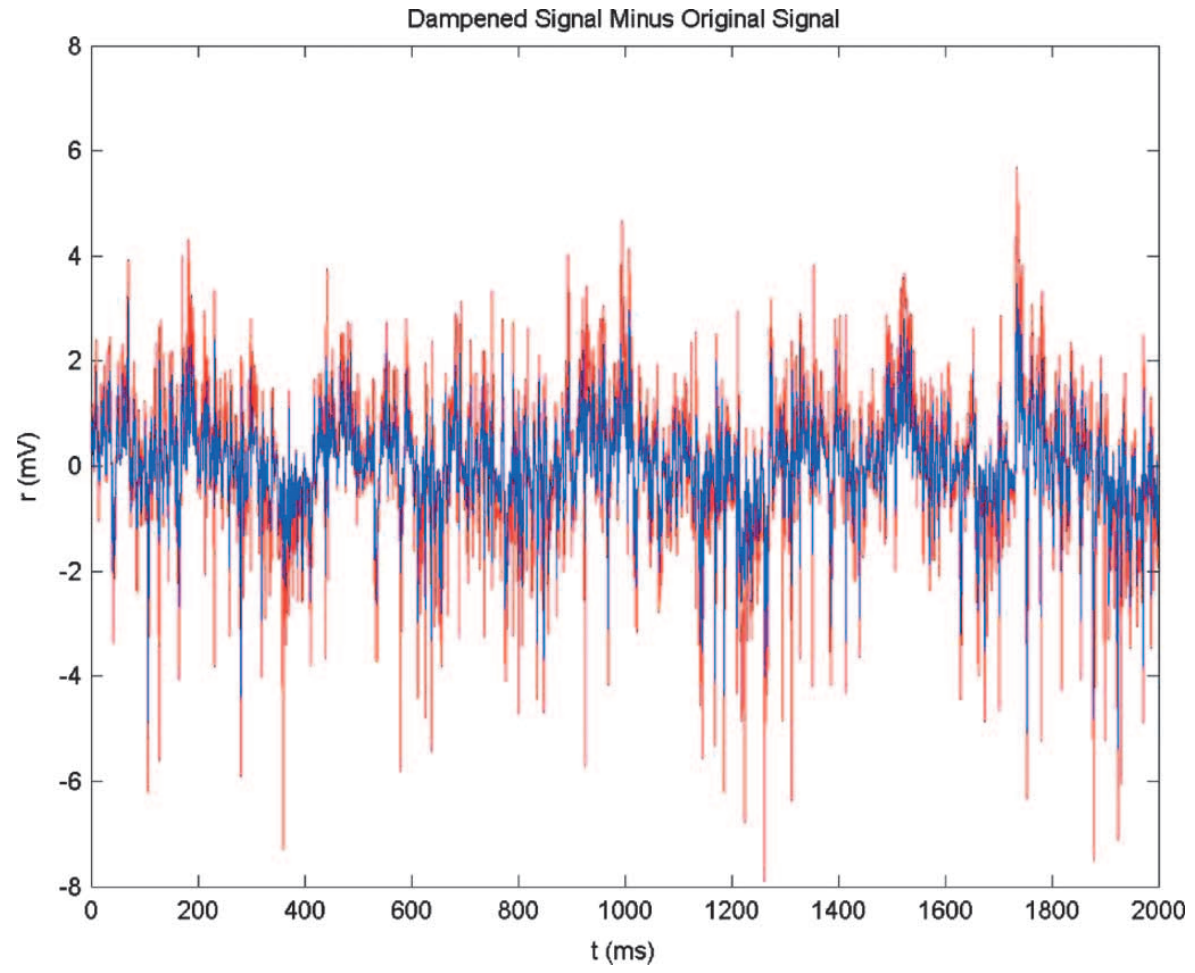

Figure 9. Superimposition of the difference between the original and the damped signals (blue) over the original one (red) for $a=0.45$ and $b=0.55$.

Table 1. ASLE has been calculated both for $x$ and $y$ directions, the larger one is to be compared with the largelyap value.

\begin{tabular}{|c|c|c|}
\hline Channel & ASLE (along $x$ and $y$ axes) & Largelyap \\
\hline 2 & $\begin{array}{l}\lambda_{x}=0.2047 \\
\lambda_{y}=0.2055\end{array}$ & $\lambda=0.2068$ \\
\hline 3 & $\begin{array}{l}\lambda_{x}=0.3501 \\
\lambda_{y}=0.3342\end{array}$ & $\lambda=0.2254$ \\
\hline 4 & $\begin{array}{l}\lambda_{x}=0.8261 \\
\lambda_{y}=0.8291\end{array}$ & $\lambda=0.8791$ \\
\hline 5 & $\begin{array}{l}\lambda_{x}=0.6772 \\
\lambda_{y}=0.6933\end{array}$ & $\lambda=0.6507$ \\
\hline 6 & $\begin{array}{l}\lambda_{x}=0.6737 \\
\lambda_{y}=0.6461\end{array}$ & $\lambda=0.6026$ \\
\hline 7 & $\begin{array}{l}\lambda_{x}=0.6661 \\
\lambda_{y}=0.6172\end{array}$ & $\lambda=0.6477$ \\
\hline 8 & $\begin{array}{l}\lambda_{x}=0.5542 \\
\lambda_{y}=0.5418\end{array}$ & $\lambda=0.5005$ \\
\hline 9 & $\begin{array}{l}\lambda_{x}=0.9258 \\
\lambda_{y}=0.9152\end{array}$ & $\lambda=0.9001$ \\
\hline 10 & $\begin{array}{l}\lambda_{x}=0.9356 \\
\lambda_{y}=0.9089\end{array}$ & $\lambda=0.4830$ \\
\hline 11 & $\begin{array}{l}\lambda_{x}=0.7125 \\
\lambda_{y}=0.7015\end{array}$ & $\lambda=0.7174$ \\
\hline
\end{tabular}


e.g. VNS works has not yet been understood fully [11]. The stimulation dosage is fixed by the physician in a stipulated step by step manner. If the patient experiences problems then the dosage is lowered. Otherwise it is maintained at a standard level [11]. Since the effect of stimulation therapy is to suppress seizure activity and thereby suppress spikes in the EEG signal, we can measure a more appropriate patient-specific dosage titration for the VNS with the help of the spike suppression algorithm described in section 2.

Once the patient is surgically implanted with the vagus nerve stimulator (Cyberonics Corporation, Houston, TX, USA) and the wound heals in about two weeks, the doctor begins the dosage titration with a non-invasive wand fitted with a computer loaded with a software according to a fixed guideline (for detail see [11]). According to our proposed protocol the patient will have to be administered mild test stimulations by the implanted stimulator within the currently stipulated limit [11]. The patient's EEG will have to be monitored before, during and immediately after the test stimulations. Let average EEG amplitude before stimulation be $r_{a b}$ and after $r_{a f}$, where subscripts $a, b$ and $f$ stand for average, before and after, respectively.

$$
r_{a b}=\frac{\int_{-T}^{0} r_{b}(t) \mathrm{d} t}{\int_{-T}^{0} \mathrm{~d} t}
$$

and

$$
r_{a f}=\frac{\int_{0}^{T^{\prime}} r_{a}(t) \mathrm{d} t}{\int_{0}^{T^{\prime}} \mathrm{d} t},
$$

where $T$ and $T^{\prime}$ are (both positive) suitable time intervals and 0 denotes the instance of stimulus onset. $I$ be the average stimulus intensity. Now consider $N$ number of trials. Let data obtained at the ith trial be indexed by $i$. Consider the sequence $\left(I^{i}, r_{a b}^{i}-r_{a f}^{i}\right)_{i=1}^{N}$. Plot these $N$ points on the Euclidean plane. Had the system worked according to Ohm's law all these points would have been arranged in a straight line passing through the origin. But it is highly unlikely that the brain under stimulation will behave like a perfect Ohmic system. In that case we can determine the least square regression line [14] with respect to these $N$ points. In case of Ohmic system this line would have been the line passing through all the $N$ points and it's slope would have given the resistance of the medium (tissues in this case). In analogy to this we take the slope of the regression line to be the value of the patient characteristic measure $P$. We propose the amount of stimulation to be applied to the brain is

$$
I(t)=\frac{\Delta r}{P},
$$

where $\Delta r$ is the amplitude suppression in the EEG signal.

Next we shall have to determine $\Delta r$ in order to determine $I(t)$ according to equation (5.3). This can be done in either of two ways. First, spikes can be detected in the patient's EEG signal during occurrence of seizure by visual inspection by the physician or by an automated spike detection algorithm $[15,16]$. Then the physician can determine by how much the percentage of that spike amplitude will have to be suppressed. $a=b$ (fixed mode method as described in section 2) is to be chosen accordingly in equation (2.1) and this will give the value of the $\Delta r$ to be substituted in equation (5.3). Alternatively, we can choose 
$a>0.5$ and $b<0.5$ (variable mode method as described in section 2) to run the spike suppression algorithm on the off line EEG signal collected from that particular patient during the onset of epileptic seizure. $a$ and $b$ are to be chosen to suppress the (off line) seizure EEG signal to a desired degree. Then these particular values of $a$ and $b$ are to be substituted in equation (2.1) for the $\Delta r$, which in turn will have to be substituted in equation (5.3) to give the stimulation current $I(t)$.

Note that frequency and amplitude of $I(t)$ are both variable since they are variable in $\Delta r(t)$. In particular frequency of $I(t)$ is equal to the frequency of $\Delta r(t)$, which is equal to the frequency of the EEG signal to be suppressed. But the frequency of the EEG signal may often be more than $30 \mathrm{~Hz}$ and this requires that according to this protocol the brain should be stimulated at a rate more than $30 \mathrm{~Hz}$, which is beyond the current limit stipulated by the Federal Drug Administration of the USA [11]. This prevents the clinical trial of this protocol. Nevertheless it indicates the importance of model based research on stimulation therapy, which may eventually lead to an improved stimulation protocol one day with better results and lesser side effects.

\section{Conclusion}

In this paper we have described a novel amplitude suppression algorithm, which can reduce the amplitudes of spikes in an epileptic EEG signal to any desired degree without altering any other characteristics of the signal. We have shown that this algorithm can also reduce chaoticity in a chaotic signal. For this purpose we had to define a new measure of chaoticity for chaotic signals and with respect to this measure the reduction of chaoticity has been achieved. The new measure has been named ASLE. We have compared the largest value of ASLE with the largest Lyapunov exponent and their close fit in most cases suggests that the largest ASLE is a good measure of chaoticity in a signal. Our experimentation in section 4 has shown that the largest ASLE may even be a better measure of chaoticity in a chaotic signal than the largest Lyapunov exponent as estimated by largelyap algorithm. Moreover ASLE is easier to compute than the Lyapunov exponent.

Apart from reducing chaoticity the spike (and amplitude) suppression algorithm has at least two other important potentialities. A new VNS protocol based on this algorithm has been outlined in this paper. This new stimulation protocol proposes a patient specific dosage titration for the VNS therapy. Contrary to the current practice it suggests a variable amplitude and frequency stimulation and frequency of the stimulation may often be more than the current FDA approved limit of $30 \mathrm{~Hz}$. Various advantages of higher frequency stimulation has been discussed in [10] and in the references there in. We have shown how the dosage titration should be controlled in a patient-specific manner for such a higher frequency stimulation.

Another potential benefit of the algorithm is that it can very efficiently amplify EEG signals by any desired degree. Raw EEG, after collection, is often amplified with the help of an electronic amplifier, which adds up noise to the signal. As discussed in section 2 , if $a, b$ are both taken to be $<1$ the amplitude is suppressed, but if they are taken $>1$ then for the same reasoning the algorithm will amplify the signal (augment the amplitude) without changing any other characteristics by the slightest amount. If at least one of them is $>1$ then also the signal will be amplified. Like the suppression the amplification will also have two modes-fixed and variable. It is clear that if condition (2.4) is followed the amplification will be in fixed mode, otherwise it will be in variable mode. By equation (2.4) the signal can be 
amplified by any fixed percentage over each time instant. Amplifier noise is an important issue in the relevant biomedical engineering industries. Since this algorithm can amplify EEG signal just by computer implementation (writing only a few tens of lines of code in MATLAB or perhaps a longer code in any other suitable language), it does not add up any noise to the already fragile data. Moreover it will considerably reduce the cost of amplification and make the apparatus for collecting the signal handy.

\section{Acknowledgements}

The work has been partially supported by a National Science Foundation grant \# NSF-EIA01-303532. KM was visiting the University of Memphis as a Fulbright Scholar, during which he has done this research. We are thankful to the Neurology Department of the University of Tennessee at Memphis for supplying the EEG data. We would also like to thank Dr B. Adamolekun of the same department for many stimulating discussions and help with literature.

\section{References}

[1] Majumdar, K. and Myers, M.H., A novel spike suppression algorithm for EEG signals and its possible therapeutic implications on epilepsy, (submitted).

[2] Ott, E., Grebogy, C. and Yorke, J.A., 1990, Controlling chaos, Physical Review Letters, 64, 1196-1199.

[3] Chen, G. and Dong, X., 1998, From Chaos to Order: Methodologies, Perspectives and Applications (Singapore: World Scientific).

[4] Devaney, R.L., 1989, An Introduction to Chaotic Dynamical Systems (Cambridge, MA: Perseus Publishing).

[5] Banks, J., Brooks, J., Cairns, G., Davis, G. and Stacey, P., 1992, On Devaney's definition of chaos, American Mathematical Monthly, 99, 332-334.

[6] Skarda, C.A. and Freeman, W.J., 1987, How brains make chaos in order to make sense of the world, Behavioral and Brain Sciences, 10, 161-195.

[7] Tsuda, I., 2001, Toward an interpretation of dynamic neural activity in terms of chaotic dynamical systems, Behavioral and Brain Sciences, 10, 793-847.

[8] Porat, B., 1996, A Course in Digital Signal Processing (New York, NY: John Wiley \& Sons).

[9] http://www.physik3.gwdg.de/tstool/HTML/node47.html.

[10] Durand, D.M. and Bikson, M., 2001, Suppression and control of epileptiform activity by electrical stimulation: a review. Proceedings of IEEE, 89, pp. 1065-1082.

[11] Heck, C., Helmers, S.L. and DeGiorgio, C.M., 2002, Vagus nerve stimulation therapy, epilepsy, and device parameters: scientific basis and recommendations for use, Neurology, 59(Suppl. 4), S31-S37.

[12] Wolf, A., Swift, J.B., Swinney, H.L. and Vastano, J.A., 1985, Determining Lyapunov exponent from a time series, Physica D, 16, 285-316.

[13] Durand, D.M. and Bikson, M., 2001, Suppression and control of epileptiform activity by electrical stimulation: a review. Proceeding of IEEE, 89, pp. 1065-1082.

[14] Hoel, P.G., Port, S.C. and Stone, C.J., 1971, Introduction to Statistical Theory (Boston: Houghton Mifflin).

[15] Bhavaraju, N.C., Frei, M.G. and Osorio, I., 2006, Analog seizure detection and performance evaluation, IEEE Transaction on Biomedical Engineering, 53, 238-245.

[16] Osorio, I., Frei, M.G. and Wilkinson, S.B., 1998, Real-time automated detection and quantitative analysis of seizures and short-term prediction of clinical onset, Epilepsia, 39, 615-627. 


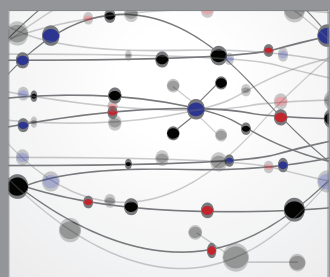

The Scientific World Journal
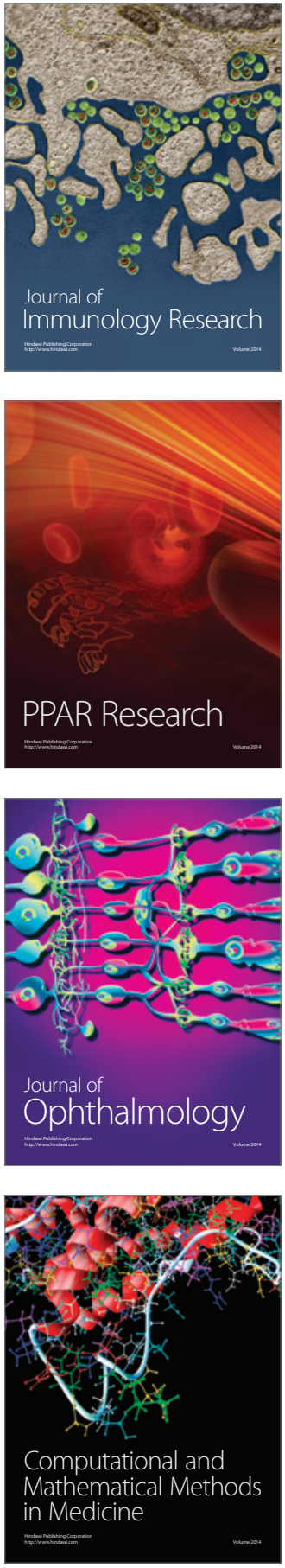

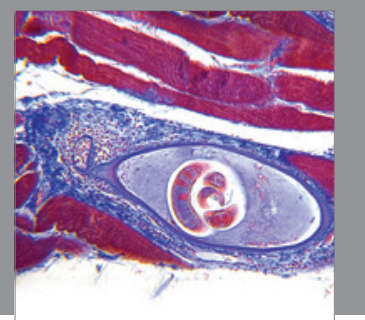

Gastroenterology

Research and Practice
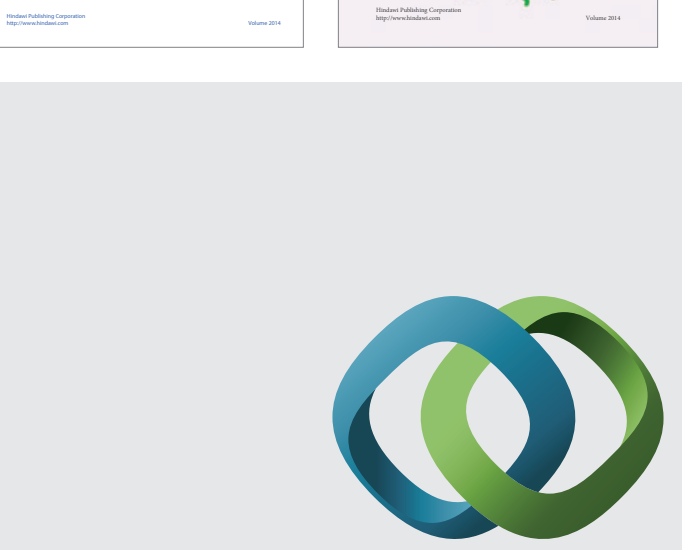

\section{Hindawi}

Submit your manuscripts at

http://www.hindawi.com
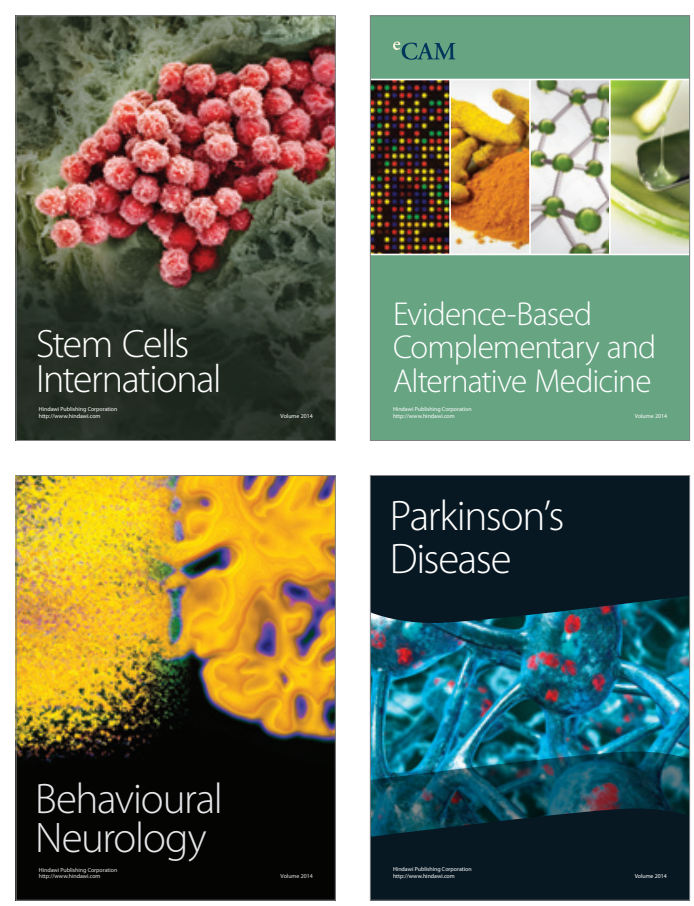

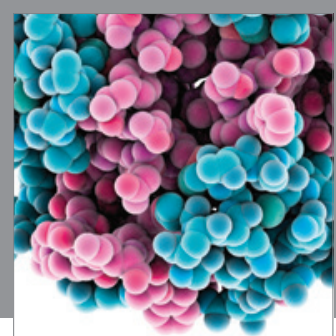

Journal of
Diabetes Research

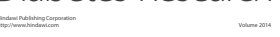

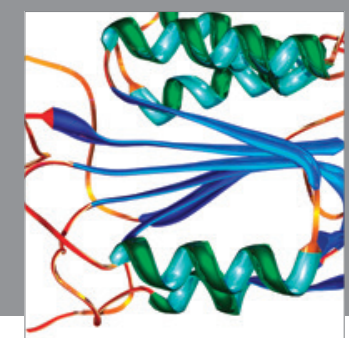

Disease Markers
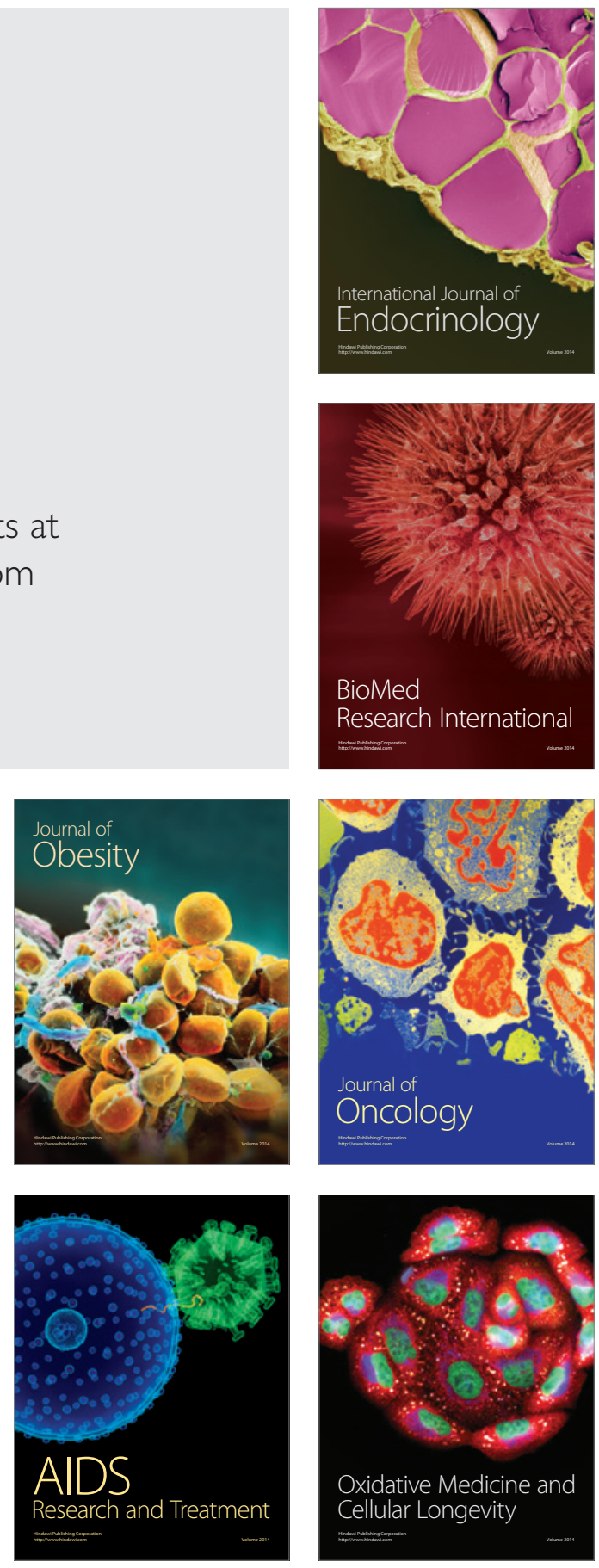